\title{
Crystal structure and computational study of an oxo-bridged bis-titanium(III) complex
}

\author{
Hannah R. Bloomfield, Joshua W. Hollett and Jamie S. Ritch
}

Acta Cryst. (2021). C77, 391-394

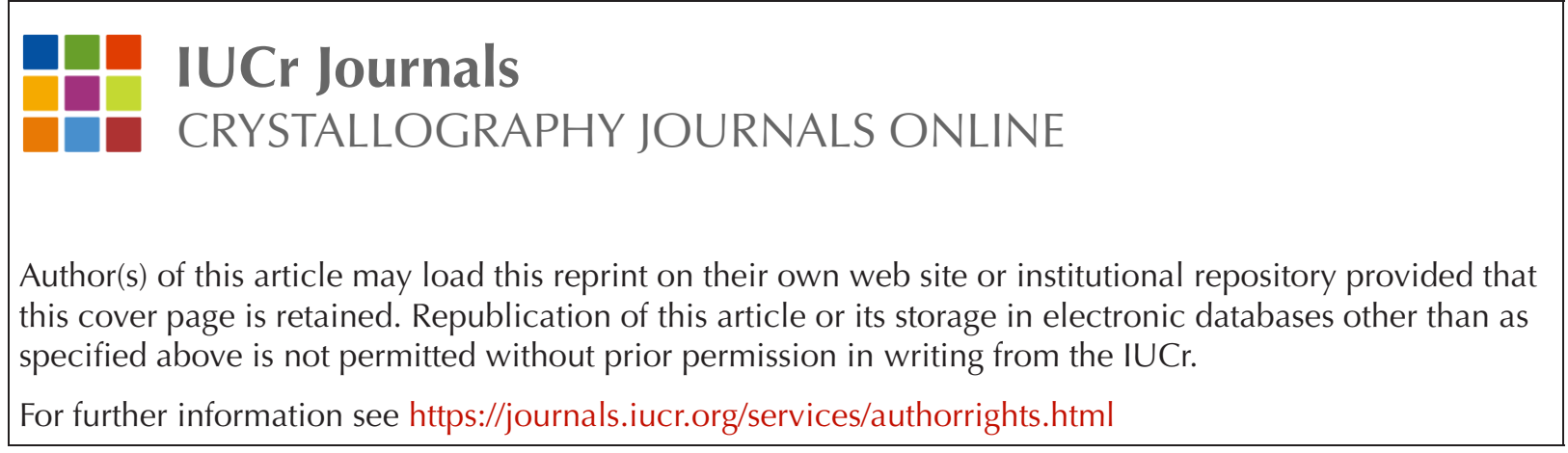




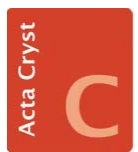

STRUCTURAL

CHEMISTRY

ISSN 2053-2296

Received 12 May 2021

Accepted 10 June 2021

Edited by R. Diniz, Universidade Federal de Minas Gerais, Brazil

Keywords: titanium(III); bimetallic complex; coordination compound; crystal structure; computational modelling.

CCDC reference: 2089304

Supporting information: this article has supporting information at journals.iucr.org/c
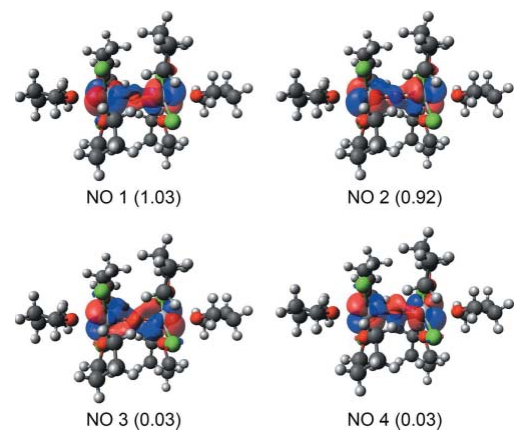

\section{Crystal structure and computational study of an oxo-bridged bis-titanium(III) complex}

\author{
Hannah R. Bloomfield, Joshua W. Hollett and Jamie S. Ritch* \\ Department of Chemistry, The University of Winnipeg, 515 Portage Avenue, Winnipeg MB R3B 2E9, Canada. \\ ${ }^{*}$ Correspondence e-mail: j.ritch@uwinnipeg.ca
}

The solid-state structure of the new compound $\mu$-oxido-bis[dichloridotris(tetrahydrofuran- $\kappa O)$ titanium(III)], $\left[\mathrm{Ti}_{2} \mathrm{Cl}_{4} \mathrm{O}\left(\mathrm{C}_{4} \mathrm{H}_{8} \mathrm{O}\right)_{6}\right]$, at $150 \mathrm{~K}$ has been determined. The crystal has monoclinic $(C 2 / c)$ symmetry and the complex features $C_{2}$ symmetry about the bridging $\mathrm{O}$ atom. Positional disorder is evident in one of the three tetrahydrofuran environments. A post-Hartree-Fock computational analysis indicates that the complex has nearly degenerate triplet and singlet spin states, with the former favoured slightly by ca $2 \mathrm{~kJ} \mathrm{~mol}^{-1}$.

\section{Introduction}

Molecular titanium oxo species are numerous, with the vast majority featuring the metal in the +4 oxidation state. In addition to their diverse structural chemistry, they can have varying applications, such as for $\mathrm{CO}_{2}$ sorption (Hong \& Chun, 2013) or as precursors to ceramics (Boyle et al., 2002) and polyoxometalates (Ishimoto et al., 2015). Amongst structurally characterized dititanium(IV) clusters with bridging oxo groups, supporting ligands include cyclopentadienyl (Luo et al., 2009) and salen [N,N'-ethylenebis(salicylimine)] ligands (Aoyama et al., 1988) or, in some cases, simply solvent molecules (Strel'tsova et al., 1988; Mahrwald et al., 2001). Given the highly oxophilic nature of titanium, in many cases these species arise from the unexpected oxidation and/or hydrolysis of a monometallic precursor complex (Crestani et al., 2013; Barroso et al., 2013).

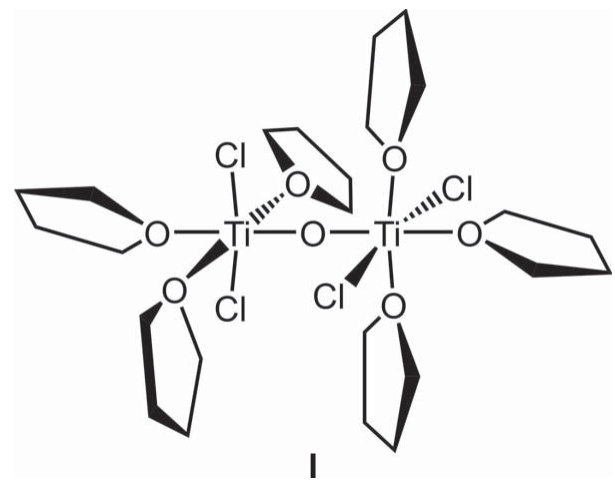

Reports of $\mathrm{Ti}^{\mathrm{III}}$ analogues with this motif are rare, though titanium(III) compounds have many practical applications, including as components in heterogeneous Ziegler-Natta alkene polymerization catalysts (Ziegler et al., 1955; Natta et $a l ., 1955)$, and as reagents/catalyts in various organic transformations (Rosales et al., 2015). Herein we report the first instance of a structurally characterized dititanium(III) oxychloride molecule, $\left[\left\{\mathrm{TiCl}_{2}(\mathrm{THF})_{3}\right\}_{2}(\mu-\mathrm{O})\right](\mathbf{1})$, presenting a structural analysis and computational modelling. 
Table 1

Experimental details.

\begin{tabular}{|c|c|}
\hline \multicolumn{2}{|l|}{ Crystal data } \\
\hline Chemical formula & {$\left[\mathrm{Ti}_{2} \mathrm{Cl}_{4} \mathrm{O}\left(\mathrm{C}_{4} \mathrm{H}_{8} \mathrm{O}\right)_{6}\right]$} \\
\hline$M_{\mathrm{r}}$ & 686.22 \\
\hline Crystal system, space group & Monoclinic, $C 2 / c$ \\
\hline Temperature $(\mathrm{K})$ & 150 \\
\hline$a, b, c(\AA)$ & 19.1347 (5), 9.1227 (3), 18.7937 (5) \\
\hline$\beta\left(^{\circ}\right)$ & $102.092(2)$ \\
\hline$V\left(\AA^{3}\right)$ & $3207.84(16)$ \\
\hline$Z$ & 4 \\
\hline Radiation type & Mo $K \alpha$ \\
\hline$\mu\left(\mathrm{mm}^{-1}\right)$ & 0.87 \\
\hline Crystal size $(\mathrm{mm})$ & $0.33 \times 0.33 \times 0.28$ \\
\hline \multicolumn{2}{|l|}{ Data collection } \\
\hline Diffractometer & Bruker APEXII CCD \\
\hline Absorption correction & $\begin{array}{l}\text { Numerical (SADABS; Bruker, } \\
\text { 2016) }\end{array}$ \\
\hline$T_{\min }, T_{\max }$ & $0.562,0.678$ \\
\hline $\begin{array}{l}\text { No. of measured, independent and } \\
\text { observed }[I>2 \sigma(I)] \text { reflections }\end{array}$ & $25791,4461,3244$ \\
\hline$R_{\text {int }}$ & 0.068 \\
\hline$(\sin \theta / \lambda)_{\max }\left(\AA^{-1}\right)$ & 0.695 \\
\hline \multicolumn{2}{|l|}{ Refinement } \\
\hline$R\left[F^{2}>2 \sigma\left(F^{2}\right)\right], w R\left(F^{2}\right), S$ & $0.073,0.138,1.13$ \\
\hline No. of reflections & 4461 \\
\hline No. of parameters & 182 \\
\hline No. of restraints & 10 \\
\hline $\mathrm{H}$-atom treatment & $\mathrm{H}$-atom parameters constrained \\
\hline$\Delta \rho_{\max }, \Delta \rho_{\min }\left(\mathrm{e} \AA^{-3}\right)$ & $0.57,-0.41$ \\
\hline
\end{tabular}

Computer programs: SAINT (Bruker, 2016), APEX2 (Bruker, 2016), SHELXT2018 (Sheldrick, 2015a), SHELXL (Sheldrick, 2015b) and OLEX2 (Dolomanov et al., 2009).

\section{Experimental}

\subsection{Synthesis and crystallization}

Manipulations were conducted under an inert atmosphere of argon using standard Schlenk techniques. Tetrahydrofuran (THF) and hexanes were dried using a solvent purification system, and stored in PTFE-stoppered flasks over activated $4 \AA$ molecular sieves. The complex $\left[\mathrm{TiCl}_{3}(\mathrm{THF})_{3}\right]$ was obtained from Strem and used as received. The compound 1,3-ditert-butylimidazolin-2-ylidene ( $\left.\mathrm{SI}{ }^{t} \mathrm{Bu}\right)$ was prepared according to a reported procedure (Denk et al., 1997).

The title compound (1) was prepared in an attempt to synthesize an N-heterocyclic carbene-ligated derivative of this $\mathrm{Ti}^{\mathrm{III}}$ complex. Complex $\mathbf{1}$ was obtained from an equimolar solution of $\left[\mathrm{TiCl}_{3}(\mathrm{THF})_{3}\right]$ and $\mathrm{SI}^{t} \mathrm{Bu}$ in THF layered with hexanes and stored at $5{ }^{\circ} \mathrm{C}$. After several weeks, a single red crystal was observed to form, which was used in subsequent diffraction studies.

\subsection{Refinement}

Crystal data, data collection and structure refinement details are summarized in Table $1 . \mathrm{H}$ atoms were placed in calculated positions $(\mathrm{C}-\mathrm{H}=0.99 \AA)$ and refined according to a riding model, with $U_{\text {iso }}(\mathrm{H})=1.2 U_{\text {eq }}(\mathrm{C})$. One THF ligand in the asymmetric unit showed signs of positional disorder and was modelled as a two-part $c a$ 70:30 anisotropic mixture, with $\mathrm{C}-\mathrm{O}$ and $\mathrm{C}-\mathrm{C}$ distances restrained to obtain reasonable geometries.

\section{Results and discussion}

A single crystal of the new complex $\left[\left\{\mathrm{TiCl}_{2}(\mathrm{THF})_{3}\right\}_{2}(\mu-\mathrm{O})\right](\mathbf{1})$ was obtained unexpectedly from a solution of the well-known $\mathrm{Ti}^{\mathrm{III}}$ salt $\left[\mathrm{TiCl}_{3}(\mathrm{THF})_{3}\right]$. Presumably, adventitious moisture caused a partial hydrolysis of this complex. Compound $\mathbf{1}$ is the first reported bimetallic dichloridotitanium(III) complex bridged by an oxo ligand. A pyridine-substituted bromide complex, $\left[\left\{\operatorname{TiBr}_{2}(\mathrm{py})_{3}(\mu-\mathrm{O})\right\}_{2}\right]$, has been reported previously (Troyanov et al., 1990).

The asymmetric unit of complex 1 contains one $\left\{\mathrm{TiCl}_{2^{-}}\right.$ $(\mathrm{THF})_{3} \mathrm{O}$ \} moiety with the oxo ligand sitting on a twofold rotational axis. Thus, the complex has ideal $C_{2}$ symmetry. The titanium centres feature distorted octahedral coordination, with the chloride ligands on each metal trans, leaving the THF and oxo ligands in an approximate square plane. The THF ligands exhibit twist conformations. The two $\mathrm{Cl}-\mathrm{Ti}-\mathrm{Cl}$ units are staggered when viewed down the $\mathrm{Ti}-\mathrm{O}-\mathrm{Ti}$ axis. As in the precursor compound $\left[\mathrm{TiCl}_{3}(\mathrm{THF})_{3}\right]$, the THF ligands are meridionally coordinated. The overall molecular structure is depicted in Fig. 1 and the packing within the unit cell is shown in Fig. 2.

The $\mathrm{Ti}-\mathrm{O}$ (oxo) distances are 1.8091 (7) $\AA$, while the Ti$\mathrm{O}$ (THF) distances range from 2.142 (3) to 2.229 (3) $\AA$, with the longest distance observed for the THF ligand trans to the strongly donating oxo group. The $\mathrm{Ti}-\mathrm{Cl}$ distances are 2.4398 (12) and $2.4482(12) \AA$. The $\mathrm{O}-\mathrm{Ti}-\mathrm{O}-\mathrm{Ti}-\mathrm{O}$ fragment containing the THF ligands trans to the oxo group is nearly linear, with $\mathrm{O}-\mathrm{Ti}-\mathrm{O}$ and $\mathrm{Ti}-\mathrm{O}-\mathrm{Ti}$ bond angles of $179.21(13)$ and $179.1(3)^{\circ}$, respectively. The ligands cis to the oxo group are bent away from it, with $\mathrm{O}-\mathrm{Ti}-X$ angles in the range $95.20(13)-97.47(9)^{\circ}$. No significant intermolecular interactions were identified in the crystal structure.

Compared to the analogous ligand values in $\left[\mathrm{TiCl}_{3}(\mathrm{THF})_{3}\right]$ (Handlovič et al., 1981), the Ti-Cl distances in complex $\mathbf{1}$ are elongated by $c a 4 \%$ [cf. 2.341 (3)-2.360 (3) $\mathrm{A}]$ and the Ti-O

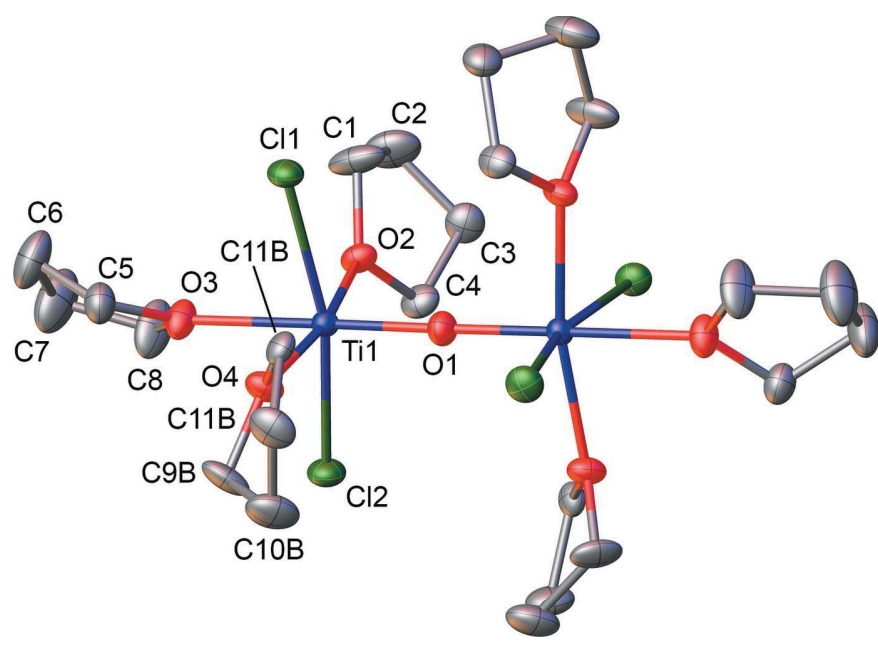

Figure 1

Displacement ellipsoid plot (50\% probability level) of complex 1. C atoms with the suffix ' $B$ ' illustrate one part of a two-part anisotropic disorder model. Symmetry-equivalent atoms (unlabelled) are generated by the operator $\left(-x+1, y,-z+\frac{3}{2}\right)$. $\mathrm{H}$ atoms have been omitted for clarity. 


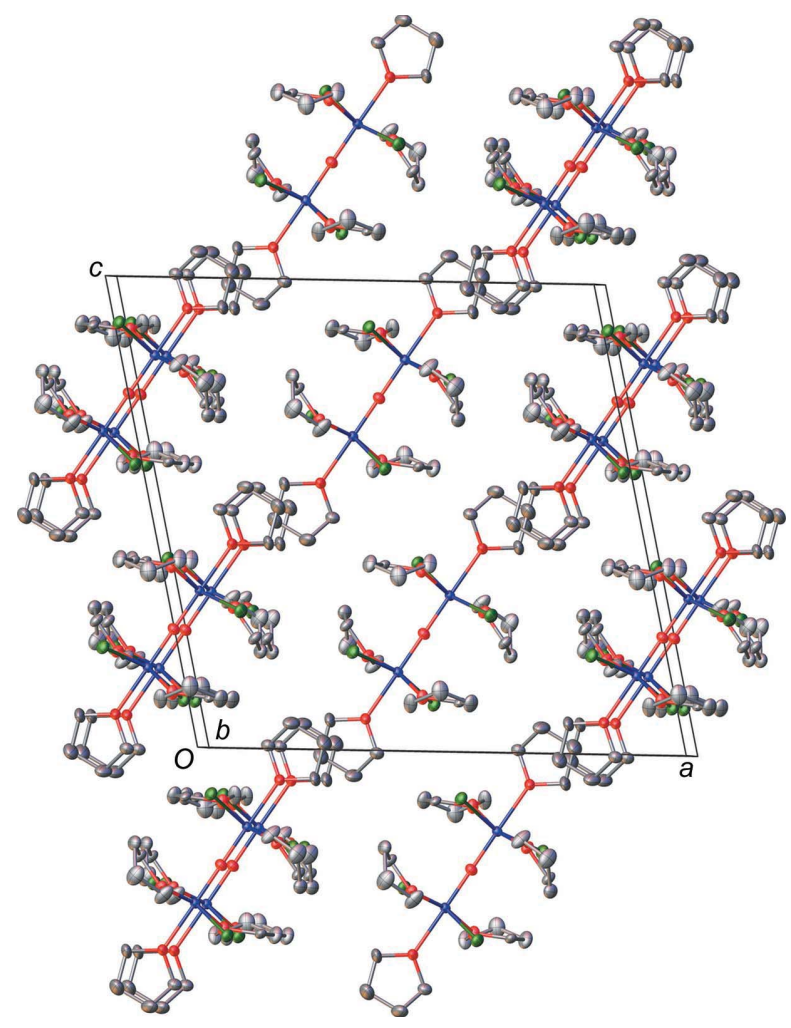

Figure 2

The packing diagram for complex $\mathbf{1}$

distances are longer by $2 \%$ [cf. 2.084 (7)-2.181 (6) $\AA$ ]. This may be reasonably ascribed to a combination of the steric influence of the two adjacent octahedral coordination environments, as well as the presence of a strongly electrondonating oxo ligand.

The related titanium(IV) complex $\left[\left\{\mathrm{TiCl}_{3}(\mathrm{THF})_{2}(\mu-\mathrm{O})\right\}_{2}\right]$ (Strel'tsova et al., 1988; Mahrwald et al., 2001) features a similar structure. It also crystallizes in the space group $C 2 / c$ with $C_{2}$ molecular symmetry. The three chloride ligands on the metal centres form mer arrangements, which are twisted relative to each other when viewed down the $\mathrm{Ti}-\mathrm{O}-\mathrm{Ti}$ direction. Compared to the title complex, the metal-ligand bond distances are shortened by $c a 1$ and $6 \%$ for $\mathrm{Ti}-\mathrm{O}$

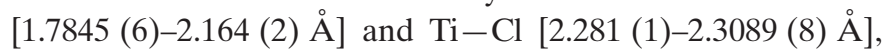
respectively, in keeping with the higher oxidation state in this $\mathrm{Ti}^{\mathrm{IV}}$ derivative. The structure of the isomorphous zirconium(IV) complex $\left[\left\{\mathrm{ZrCl}_{3}(\mathrm{THF})_{2}(\mu-\mathrm{O})\right\}_{2}\right]$ has also been reported (Guan et al., 1999). A trimetallic $\mathrm{Ti}^{\mathrm{IV}}$ analogue, $\left[\mathrm{TiCl}_{2}(\mu-\mathrm{O})\right]_{3}$, has been observed to crystallize in two different stereoisomeric forms (Gritzo et al., 2004; Zhang et al., 2014).

The vanadium(III) complex $\left[\left\{\mathrm{VCl}_{2}(\mathrm{THF})_{3}\right\}_{2}(\mu-\mathrm{O})\right]$ (Chandrasekhar \& Bird, 1984) is isomorphous with complex 1. In that case, the authors surmized that an initially formed $\mathrm{V}^{\mathrm{II}}$ species was oxidized by adventitious oxygen before forming the bimetallic complex. In this vanadium complex, the metalligand distances are $c a 2 \%$ shorter for chloride [2.384 (6)2.390 (6) $\AA$ ] and $\mu$-oxo [1.769 (5) $\AA$ ], and $c a 1 \%$ elonged for the THF ligands [2.16 (1)-2.26 (1) A] , compared to complex 1.
Since complex 1 features two adjacent $\mathrm{Ti}^{\mathrm{III}}\left(d^{1}\right)$ centres, there is the question of magnetic coupling between the two. Some experimental measurements on molecules containing the $\left[\mathrm{Ti}^{\mathrm{III}}-\mathrm{O}-\mathrm{Ti}^{\mathrm{III}}\right]$ fragment have been reported previously. For the putative complex $\left[L_{2} \mathrm{Ti}_{2} \mathrm{Cl}_{4} \mathrm{O}\right](L=1,4,7$-trimethyl1,4,7-triazacyclononane), a temperature-independent magnetic moment of $1.72 \mu_{\mathrm{B}}$ per Ti centre indicated paramagnetic behaviour, i.e. uncoupled spins, in the range 90-298 K (Bodner et al., 1990). Two related pseudohalide complexes $\left[L_{2} \mathrm{Ti}_{2} X_{4} \mathrm{O}\right]$ ( $X=\mathrm{NCO}$ or NCS) showed weak antiferromagnetic coupling, with the magnetic moment decreasing significantly from ambient temperature values of $\sim 1.7$ to $1.12-1.15 \mu_{\mathrm{B}}$ below $30 \mathrm{~K}$ (Jeske et al., 1994).

The title complex was analysed with density functional and multireference post-Hartree-Fock methods using the ORCA electronic structure package (Version 4.2.1; Neese et al., 2020). The geometries of the complex in the lowest singlet and triplet electronic states were optimized with the TPSSh density functional (Staroverov et al., 2003) and the def2-TZVP basis set (Weigend \& Ahlrichs, 2005; Hellweg et al., 2007). The optimized geometries and energies of the triplet and brokensymmetry (unrestricted) singlet states were found to be nearly equivalent, with a difference in electronic energy of $0.2 \mathrm{~kJ} \mathrm{~mol}^{-1}$ and an r.m.s. deviation with respect to the atomic coordinates of $0.002 \AA$. Therefore, subsequent calculations were performed on the TPSSh/def2-TZVP optimized triplet structure.

Taking the active electrons to be the unpaired $\alpha$ electrons and the active orbitals to be the occupied open-shell orbitals and two unoccupied orbitals, the $(2,4)$ complete-active space self-consistent field (CASSCF) wave function was determined with the same basis set. The resulting natural orbitals and their occupancies are shown in Fig. 3. The CASSCF energies were improved by applying fully internally contracted second-order $\mathrm{N}$-electron valence state perturbation theory (FIC-NEVPT2;
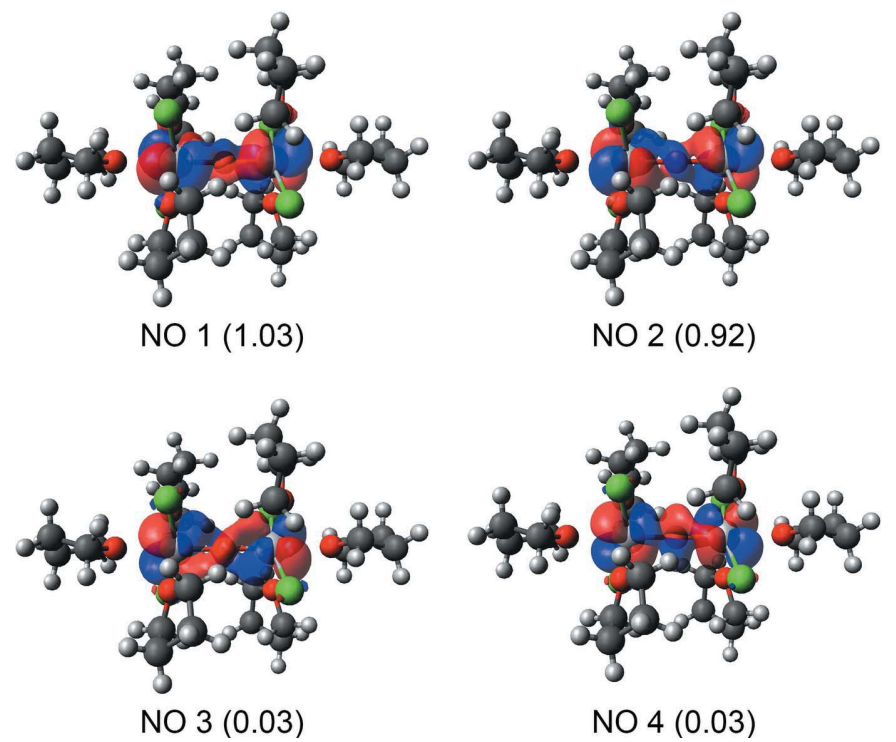

Figure 3

Natural orbitals (NOs) with occupancies shown in parenthesis for complex 1 at the $(2,4)$-CASSCF/def2-TZVP level. 
Angeli et al., 2001a,b, 2002). The FIC-NEVPT2 energies, for the $(2,4)$-CASSCF reference, of the singlet and triplet states are nearly equivalent. The triplet is predicted to be lower in energy than the singlet, by $2.4 \mathrm{~kJ} \mathrm{~mol}^{-1}$. These findings are thus generally consistent with previously discussed experimental, as well as prior configuration interaction (CI) studies (Fink et al., 1994), which indicate no strong coupling of electron spins in linear $\left[\mathrm{Ti}^{\mathrm{III}}-\mathrm{O}-\mathrm{Ti}^{\mathrm{III}}\right]$ systems. One difference is the finding that the triplet state is slightly more stable in complex 1, which would predict ferromagnetic coupling at low temperatures.

Experimental confirmation of the magnetic behaviour of $\mathbf{1}$, along with other spectroscopic properties, will require further study on additional quantities of the complex. An intentional synthesis should be possible via controlled hydrolysis of the precursor $\left[\mathrm{TiCl}_{3}(\mathrm{THF})_{3}\right]$.

\section{Acknowledgements}

We thank Dr David Herbert (Department of Chemistry, University of Manitoba) for access to a single-crystal X-ray diffractometer.

\section{Funding information}

Funding for this research was provided by: Natural Sciences and Engineering Research Council of Canada (grant No. RGPIN-2019-06725).

\section{References}

Angeli, C., Cimiraglia, R., Evangelisti, S., Leininger, T. \& Malrieu, J.-P. (2001a). J. Chem. Phys. 114, 10252-10264.

Angeli, C., Cimiraglia, R. \& Malrieu, J.-P. (2001b). Chem. Phys. Lett. 350, 297-305.

Angeli, C., Cimiraglia, R. \& Malrieu, J.-P. (2002). J. Chem. Phys. 117, 9138-9153.

Aoyama, T., Ohba, S., Saito, Y., Sasaki, C., Kojima, M., Fujita, J. \& Nakajima, K. (1988). Acta Cryst. C44, 1309-1311.

Barroso, S., Madeira, F., Calhorda, M. J., Ferreira, M. J., Duarte, M. T. \& Martins, A. M. (2013). Inorg. Chem. 52, 9427-9439.

Bodner, A., Drueke, S., Wieghardt, K., Nuber, B. \& Weiss, J. (1990). Angew. Chem. Int. Ed. Engl. 29, 68-70.

Boyle, T. J., Zechmann, C. A., Alam, T. M., Rodriguez, M. A., Hijar, C. A. \& Scott, B. L. (2002). Inorg. Chem. 41, 946-957.
Bruker (2016). APEX2, SAINT and SADABS. Bruker AXS Inc., Madison, Wisconsin, USA.

Chandrasekhar, P. \& Bird, P. H. (1984). Inorg. Chem. 23, 3677-3679.

Crestani, M. G., Hickey, A. K., Gao, X., Pinter, B., Cavaliere, V. N., Ito, J.-I., Chen, C.-H. \& Mindiola, D. (2013). J. Am. Chem. Soc. 135, 14754-14767.

Denk, M. K., Thadani, A., Hatano, K. \& Lough, A. J. (1997). Angew. Chem. Int. Ed. Engl. 36, 2607-2609.

Dolomanov, O. V., Bourhis, L. J., Gildea, R. J., Howard, J. A. K. \& Puschmann, H. (2009). J. Appl. Cryst. 42, 339-341.

Fink, K., Fink, R. \& Staemmler, V. (1994). Inorg. Chem. 33, 62196229.

Gritzo, H., Schaper, F. \& Brintzingerm, H.-H. (2004). CSD Communication. Refcode IQOTAJ. CCDC, Cambridge, England.

Guan, J., Siebel, E. \& Fischer, R. D. (1999). Acta Cryst. C55, 14491451.

Handlovič, M., Mikloš, D. \& Zikmund, M. (1981). Acta Cryst. B37, 811-814.

Hellweg, A., Hättig, C., Höfener, S. \& Klopper, W. (2007). Theor. Chem. Acc. 117, 587-597.

Hong, K. \& Chun, H. (2013). Inorg. Chem. 52, 9705-9707.

Ishimoto, R., Kamata, K., Suzuki, K., Yamaguchi, K. \& Mizuno, N. (2015). Dalton Trans. 44, 10947-10951.

Jeske, P., Wieghardt, K. \& Nuber, B. (1994). Inorg. Chem. 33, 47-53.

Luo, S., Shen, B., Li, B., Song, H., Xu, H. \& Wang, B. (2009). Organometallics, 28, 3109-3112.

Mahrwald, R., Ziemer, B. \& Troyanov, S. (2001). Tetrahedron Lett. 42 , 6843-6845.

Natta, G., Pino, P., Corradini, P., Danusso, F., Mantica, E., Mazzanti, G. \& Moraglio, G. (1955). J. Am. Chem. Soc. 77, 1708-1710.

Neese, F., Wennmohs, F., Becker, U. \& Riplinger, C. (2020). J. Chem. Phys. 152, 224108.

Rosales, A., Rodríguez-García, I., Muñoz-Bascón, J., Roldan-Molina, E., Padial, N. M., Morales, L. P., García-Ocaña, M. \& Oltra, J. E. (2015). Eur. J. Org. Chem. 2015, 4567-4591.

Sheldrick, G. M. (2015a). Acta Cryst. A71, 3-8.

Sheldrick, G. M. (2015b). Acta Cryst. C71, 3-8.

Staroverov, V., Scuseria, G., Tao, J. \& Perdew, J. (2003). J. Chem. Phys. 119, 12129-12137.

Strel'tsova, N. R., Ivakina, L. V., Bel'skii, V. K., Storozhenko, P. A. \& Bulichev, B. M. (1988). Koord. Khim. 14, 421-425.

Troyanov, S. I., Mazo, G. N., Rybakov, V. B. \& Budkina, K. V. (1990). Koord. Khim. 16, 466-476.

Weigend, F. \& Ahlrichs, R. (2005). Phys. Chem. Chem. Phys. 7, 32973305.

Zhang, L., Yu, X., Zhang, L., Zhou, X. \& Lin, Y. (2014). Org. Chem. Front. 1, 929-935.

Ziegler, K., Holzkamp, E., Breil, H. \& Martin, H. (1955). Angew. Chem. 67, 541-547. 


\section{supporting information}

Acta Cryst. (2021). C77, 391-394 [https://doi.org/10.1107/S2053229621006094]

\section{Crystal structure and computational study of an oxo-bridged bis-titanium(III) complex}

\section{Hannah R. Bloomfield, Joshua W. Hollett and Jamie S. Ritch}

Computing details

Data collection: SAINT (Bruker, 2016); cell refinement: APEX2 (Bruker, 2016); data reduction: SAINT (Bruker, 2016); program(s) used to solve structure: SHELXT2018 (Sheldrick, 2015a); program(s) used to refine structure: SHELXL (Sheldrick, 2015b); molecular graphics: OLEX2 (Dolomanov et al., 2009); software used to prepare material for publication: OLEX2 (Dolomanov et al., 2009).

$\mu$-Oxido-bis[dichloridotris(tetrahydrofuran- $\kappa O$ ) titanium(III)]

Crystal data

$\left[\mathrm{Ti}_{2} \mathrm{Cl}_{4} \mathrm{O}\left(\mathrm{C}_{4} \mathrm{H}_{8} \mathrm{O}\right)_{6}\right]$

$M_{r}=686.22$

Monoclinic, $C 2 / c$

$a=19.1347(5) \AA$

$b=9.1227(3) \AA$

$c=18.7937(5) \AA$

$\beta=102.092(2)^{\circ}$

$V=3207.84(16) \AA^{3}$

$Z=4$

\section{Data collection}

Bruker APEXII CCD diffractometer

$\varphi$ and $\omega$ scans

Absorption correction: numerical

(SADABS; Bruker, 2016)

$T_{\min }=0.562, T_{\max }=0.678$

25791 measured reflections

Refinement

Refinement on $F^{2}$

Least-squares matrix: full

$R\left[F^{2}>2 \sigma\left(F^{2}\right)\right]=0.073$

$w R\left(F^{2}\right)=0.138$

$S=1.13$

4461 reflections

182 parameters

10 restraints

Hydrogen site location: inferred from neighbouring sites
$F(000)=1440$

$D_{\mathrm{x}}=1.421 \mathrm{Mg} \mathrm{m}^{-3}$

Mo $K \alpha$ radiation, $\lambda=0.71073 \AA$

Cell parameters from 9986 reflections

$\theta=2.5-29.6^{\circ}$

$\mu=0.87 \mathrm{~mm}^{-1}$

$T=150 \mathrm{~K}$

Block, clear light red

$0.33 \times 0.33 \times 0.28 \mathrm{~mm}$

4461 independent reflections 3244 reflections with $I>2 \sigma(I)$

$R_{\text {int }}=0.068$

$\theta_{\max }=29.6^{\circ}, \theta_{\min }=2.5^{\circ}$

$h=-21 \rightarrow 25$

$k=-11 \rightarrow 12$

$l=-25 \rightarrow 26$

$\mathrm{H}$-atom parameters constrained $w=1 /\left[\sigma^{2}\left(F_{\mathrm{o}}{ }^{2}\right)+30.5697 P\right]$ where $P=\left(F_{\mathrm{o}}{ }^{2}+2 F_{\mathrm{c}}{ }^{2}\right) / 3$

$(\Delta / \sigma)_{\max }=0.001$

$\Delta \rho_{\max }=0.57 \mathrm{e} \AA^{-3}$

$\Delta \rho_{\min }=-0.41$ e $\AA^{-3}$

Extinction correction: SHELXT2018 (Sheldrick, 2015a), $\mathrm{Fc}^{*}=\mathrm{kFc}\left[1+0.001 \mathrm{xFc}^{2} \lambda^{3} / \sin (2 \theta)\right]^{-1 / 4}$

Extinction coefficient: 0.00039 (7) 


\section{Special details}

Geometry. All esds (except the esd in the dihedral angle between two 1.s. planes) are estimated using the full covariance matrix. The cell esds are taken into account individually in the estimation of esds in distances, angles and torsion angles; correlations between esds in cell parameters are only used when they are defined by crystal symmetry. An approximate (isotropic) treatment of cell esds is used for estimating esds involving 1.s. planes.

Fractional atomic coordinates and isotropic or equivalent isotropic displacement parameters $\left(\AA^{2}\right)$

\begin{tabular}{|c|c|c|c|c|c|}
\hline & $x$ & $y$ & $z$ & $U_{\text {iso }} * / U_{\text {eq }}$ & Occ. $(<1)$ \\
\hline Til & $0.43130(4)$ & $0.55202(7)$ & $0.66762(4)$ & $0.01715(16)$ & \\
\hline $\mathrm{Cl1}$ & $0.48830(5)$ & $0.37747(11)$ & $0.59944(5)$ & $0.0254(2)$ & \\
\hline $\mathrm{Cl} 2$ & $0.35094(6)$ & $0.72586(11)$ & $0.70921(6)$ & $0.0283(2)$ & \\
\hline $\mathrm{O} 1$ & 0.500000 & $0.5536(5)$ & 0.750000 & $0.0249(8)$ & \\
\hline $\mathrm{O} 2$ & $0.37164(15)$ & $0.3758(3)$ & $0.70116(15)$ & $0.0231(6)$ & \\
\hline $\mathrm{O} 3$ & $0.34579(14)$ & $0.5523(4)$ & $0.56683(14)$ & $0.0277(6)$ & \\
\hline $\mathrm{C} 1$ & $0.3783(3)$ & $0.2223(5)$ & $0.6840(3)$ & $0.0441(13)$ & \\
\hline H1A & 0.428225 & 0.188668 & 0.700561 & $0.052 *$ & \\
\hline H1B & 0.363459 & 0.205566 & 0.630934 & $0.052^{*}$ & \\
\hline $\mathrm{C} 2$ & $0.3295(3)$ & $0.1431(6)$ & $0.7241(3)$ & $0.0481(14)$ & \\
\hline $\mathrm{H} 2 \mathrm{~A}$ & 0.344622 & 0.040137 & 0.734186 & $0.057^{*}$ & \\
\hline $\mathrm{H} 2 \mathrm{~B}$ & 0.279355 & 0.145037 & 0.696499 & $0.057^{*}$ & \\
\hline $\mathrm{C} 3$ & $0.3384(2)$ & $0.2311(5)$ & $0.7932(3)$ & $0.0324(10)$ & \\
\hline $\mathrm{H} 3 \mathrm{~A}$ & 0.383188 & 0.204980 & 0.827718 & $0.038^{*}$ & \\
\hline H3B & 0.297459 & 0.216653 & 0.817106 & $0.038^{*}$ & \\
\hline $\mathrm{C} 4$ & $0.3409(2)$ & $0.3859(5)$ & $0.7659(2)$ & $0.0278(9)$ & \\
\hline $\mathrm{H} 4 \mathrm{~A}$ & 0.292234 & 0.428372 & 0.753550 & $0.033^{*}$ & \\
\hline $\mathrm{H} 4 \mathrm{~B}$ & 0.371007 & 0.448157 & 0.803364 & $0.033^{*}$ & \\
\hline $\mathrm{C} 5$ & $0.3560(2)$ & $0.5794(5)$ & $0.4940(2)$ & $0.0325(10)$ & \\
\hline H5A & 0.403410 & 0.544106 & 0.488285 & $0.038^{*}$ & \\
\hline H5B & 0.352068 & 0.685421 & 0.482495 & $0.038^{*}$ & \\
\hline $\mathrm{C} 6$ & $0.2973(3)$ & $0.4953(7)$ & $0.4456(3)$ & $0.0513(15)$ & \\
\hline H6A & 0.283008 & 0.542792 & 0.397404 & $0.061^{*}$ & \\
\hline H6B & 0.312242 & 0.393259 & 0.438912 & $0.061 *$ & \\
\hline $\mathrm{C} 7$ & $0.2381(3)$ & $0.4994(9)$ & $0.4857(3)$ & $0.0617(18)$ & \\
\hline H7A & 0.205144 & 0.581543 & 0.468189 & $0.074^{*}$ & \\
\hline H7B & 0.210670 & 0.406647 & 0.478715 & $0.074^{*}$ & \\
\hline $\mathrm{C} 8$ & $0.2718(2)$ & $0.5198(7)$ & $0.5631(3)$ & 0.0475 (14) & \\
\hline H8A & 0.248572 & 0.601541 & 0.584008 & $0.056^{*}$ & \\
\hline H8B & 0.266981 & 0.429577 & 0.590949 & $0.056^{*}$ & \\
\hline $\mathrm{O} 4 \mathrm{~A}$ & $0.47581(15)$ & $0.7265(3)$ & $0.61486(16)$ & $0.0256(6)$ & $0.305(10)$ \\
\hline C9A & $0.4666(11)$ & $0.8798(15)$ & $0.6335(10)$ & $0.037(2)$ & $0.305(10)$ \\
\hline H9AA & 0.490493 & 0.901398 & 0.684430 & $0.044^{*}$ & $0.305(10)$ \\
\hline H9AB & 0.415424 & 0.906631 & 0.625989 & $0.044^{*}$ & $0.305(10)$ \\
\hline $\mathrm{C} 10 \mathrm{~A}$ & $0.5028(10)$ & $0.9567(17)$ & $0.5800(12)$ & $0.046(2)$ & $0.305(10)$ \\
\hline $\mathrm{H} 10 \mathrm{~A}$ & 0.471578 & 0.958661 & 0.530799 & $0.055^{*}$ & $0.305(10)$ \\
\hline H10B & 0.515873 & 1.058390 & 0.595679 & $0.055^{*}$ & $0.305(10)$ \\
\hline C11A & $0.5679(11)$ & $0.866(3)$ & $0.5809(18)$ & 0.038 & $0.305(10)$ \\
\hline H11A & 0.607203 & 0.893953 & 0.621795 & $0.045^{*}$ & $0.305(10)$ \\
\hline
\end{tabular}




$\begin{array}{llllll}\text { H11B } & 0.584567 & 0.875524 & 0.534694 & 0.045^{*} & 0.305(10) \\ \text { C12A } & 0.5431(12) & 0.711(2) & 0.591(2) & 0.023(2) & 0.305(10) \\ \text { H12A } & 0.535634 & 0.656542 & 0.543999 & 0.027^{*} & 0.305(10) \\ \text { H12B } & 0.578690 & 0.657804 & 0.627323 & 0.027^{*} & 0.305(10) \\ \text { O4B } & 0.47581(15) & 0.7265(3) & 0.61486(16) & 0.0256(6) & 0.695(10) \\ \text { C9B } & 0.4520(4) & 0.8769(7) & 0.6009(5) & 0.037(2) & 0.695(10) \\ \text { H9BA } & 0.414616 & 0.902429 & 0.628045 & 0.044^{*} & 0.695(10) \\ \text { H9BB } & 0.433408 & 0.893978 & 0.548355 & 0.044^{*} & 0.695(10) \\ \text { C10B } & 0.5197(4) & 0.9630(8) & 0.6284(6) & 0.046(2) & 0.695(10) \\ \text { H10C } & 0.516303 & 1.062613 & 0.607030 & 0.055^{*} & 0.695(10) \\ \text { H10D } & 0.530015 & 0.971064 & 0.682082 & 0.055^{*} & 0.695(10) \\ \text { C11B } & 0.5756(5) & 0.8740(11) & 0.6031(6) & 0.038(3) & 0.695(10) \\ \text { H11C } & 0.622887 & 0.886866 & 0.636076 & 0.045^{*} & 0.695(10) \\ \text { H11D } & 0.579341 & 0.902179 & 0.553189 & 0.045^{*} & 0.695(10) \\ \text { C12B } & 0.5498(4) & 0.7179(10) & 0.6051(7) & 0.023(2) & 0.695(10) \\ \text { H12C } & 0.551874 & 0.666786 & 0.559096 & 0.027^{*} & 0.695(10) \\ \text { H12D } & 0.579946 & 0.663704 & 0.645962 & 0.027^{*} & 0.695(10)\end{array}$

Atomic displacement parameters $\left(\AA^{2}\right)$

\begin{tabular}{lllllll}
\hline & $U^{11}$ & $U^{22}$ & $U^{33}$ & $U^{12}$ & $U^{13}$ & $U^{23}$ \\
\hline Ti1 & $0.0167(3)$ & $0.0163(3)$ & $0.0178(3)$ & $0.0004(3)$ & $0.0021(2)$ & $-0.0008(3)$ \\
C11 & $0.0277(5)$ & $0.0219(5)$ & $0.0287(5)$ & $0.0048(4)$ & $0.0107(4)$ & $-0.0014(4)$ \\
C12 & $0.0296(5)$ & $0.0248(5)$ & $0.0323(5)$ & $0.0067(4)$ & $0.0103(4)$ & $-0.0038(4)$ \\
O1 & $0.022(2)$ & $0.028(2)$ & $0.0226(19)$ & 0.000 & $0.0002(15)$ & 0.000 \\
O2 & $0.0273(15)$ & $0.0185(14)$ & $0.0259(14)$ & $-0.0053(11)$ & $0.0108(12)$ & $-0.0033(11)$ \\
O3 & $0.0183(14)$ & $0.0435(18)$ & $0.0207(14)$ & $0.0014(13)$ & $0.0033(11)$ & $-0.0041(13)$ \\
C1 & $0.068(4)$ & $0.021(2)$ & $0.055(3)$ & $-0.010(2)$ & $0.040(3)$ & $-0.012(2)$ \\
C2 & $0.059(4)$ & $0.027(3)$ & $0.065(4)$ & $-0.014(2)$ & $0.028(3)$ & $-0.007(2)$ \\
C3 & $0.031(2)$ & $0.031(2)$ & $0.035(2)$ & $-0.0086(19)$ & $0.0091(19)$ & $0.0018(19)$ \\
C4 & $0.032(2)$ & $0.029(2)$ & $0.027(2)$ & $-0.0024(18)$ & $0.0164(18)$ & $-0.0025(17)$ \\
C5 & $0.032(2)$ & $0.040(3)$ & $0.024(2)$ & $0.002(2)$ & $0.0020(17)$ & $0.0023(19)$ \\
C6 & $0.048(3)$ & $0.078(4)$ & $0.026(2)$ & $-0.019(3)$ & $0.004(2)$ & $-0.011(3)$ \\
C7 & $0.034(3)$ & $0.105(5)$ & $0.043(3)$ & $-0.012(3)$ & $0.000(2)$ & $-0.017(3)$ \\
C8 & $0.017(2)$ & $0.089(4)$ & $0.034(3)$ & $-0.007(2)$ & $0.0015(18)$ & $-0.008(3)$ \\
O4A & $0.0231(15)$ & $0.0179(14)$ & $0.0363(17)$ & $-0.0006(11)$ & $0.0075(12)$ & $0.0034(12)$ \\
C9A & $0.031(4)$ & $0.020(3)$ & $0.060(6)$ & $0.009(2)$ & $0.012(4)$ & $0.017(4)$ \\
C10A & $0.046(4)$ & $0.021(3)$ & $0.070(6)$ & $-0.004(3)$ & $0.013(4)$ & $0.000(4)$ \\
C11A & $0.037(3)$ & $0.030(3)$ & $0.050(8)$ & $-0.005(2)$ & $0.017(4)$ & $0.012(4)$ \\
C12A & $0.024(3)$ & $0.029(2)$ & $0.016(6)$ & $-0.001(2)$ & $0.007(3)$ & $-0.004(3)$ \\
O4B & $0.0231(15)$ & $0.0179(14)$ & $0.0363(17)$ & $-0.0006(11)$ & $0.0075(12)$ & $0.0034(12)$ \\
C9B & $0.031(4)$ & $0.020(3)$ & $0.060(6)$ & $0.009(2)$ & $0.012(4)$ & $0.017(4)$ \\
C10B & $0.046(4)$ & $0.021(3)$ & $0.070(6)$ & $-0.004(3)$ & $0.013(4)$ & $0.000(4)$ \\
C11B & $0.037(3)$ & $0.030(3)$ & $0.050(8)$ & $-0.005(2)$ & $0.017(4)$ & $0.012(4)$ \\
C12B & $0.024(3)$ & $0.029(2)$ & $0.016(6)$ & $-0.001(2)$ & $0.007(3)$ & $-0.004(3)$ \\
& & & & & & \\
\hline & & & & & &
\end{tabular}


Geometric parameters ( $\left.A,{ }^{\circ}\right)$

\begin{tabular}{|c|c|c|c|}
\hline $\mathrm{Ti1}-\mathrm{C} 11$ & $2.4398(12)$ & $\mathrm{C} 7-\mathrm{C} 8$ & $1.474(7)$ \\
\hline $\mathrm{Ti} 1-\mathrm{Cl} 2$ & $2.4482(12)$ & $\mathrm{C} 8-\mathrm{H} 8 \mathrm{~A}$ & 0.9900 \\
\hline $\mathrm{Ti1}-\mathrm{O} 1$ & $1.8091(7)$ & $\mathrm{C} 8-\mathrm{H} 8 \mathrm{~B}$ & 0.9900 \\
\hline $\mathrm{Ti} 1-\mathrm{O} 2$ & $2.142(3)$ & $\mathrm{O} 4 \mathrm{~A}-\mathrm{C} 9 \mathrm{~A}$ & $1.462(14)$ \\
\hline $\mathrm{Ti1}-\mathrm{O} 3$ & $2.229(3)$ & $\mathrm{O} 4 \mathrm{~A}-\mathrm{C} 12 \mathrm{~A}$ & $1.460(14)$ \\
\hline Til-O4A & $2.144(3)$ & C9A-H9AA & 0.9900 \\
\hline Ti1-O4B & $2.144(3)$ & C9A-H9AB & 0.9900 \\
\hline $\mathrm{O} 2-\mathrm{C} 1$ & $1.448(5)$ & $\mathrm{C} 9 \mathrm{~A}-\mathrm{C} 10 \mathrm{~A}$ & 1.509 (16) \\
\hline $\mathrm{O} 2-\mathrm{C} 4$ & $1.462(4)$ & $\mathrm{C} 10 \mathrm{~A}-\mathrm{H} 10 \mathrm{~A}$ & 0.9900 \\
\hline $\mathrm{O} 3-\mathrm{C} 5$ & $1.444(5)$ & $\mathrm{C} 10 \mathrm{~A}-\mathrm{H} 10 \mathrm{~B}$ & 0.9900 \\
\hline $\mathrm{O} 3-\mathrm{C} 8$ & $1.434(5)$ & $\mathrm{C} 10 \mathrm{~A}-\mathrm{C} 11 \mathrm{~A}$ & $1.496(16)$ \\
\hline $\mathrm{C} 1-\mathrm{H} 1 \mathrm{~A}$ & 0.9900 & $\mathrm{C} 11 \mathrm{~A}-\mathrm{H} 11 \mathrm{~A}$ & 0.9900 \\
\hline $\mathrm{C} 1-\mathrm{H} 1 \mathrm{~B}$ & 0.9900 & $\mathrm{C} 11 \mathrm{~A}-\mathrm{H} 11 \mathrm{~B}$ & 0.9900 \\
\hline $\mathrm{C} 1-\mathrm{C} 2$ & $1.502(7)$ & $\mathrm{C} 11 \mathrm{~A}-\mathrm{C} 12 \mathrm{~A}$ & $1.511(15)$ \\
\hline $\mathrm{C} 2-\mathrm{H} 2 \mathrm{~A}$ & 0.9900 & $\mathrm{C} 12 \mathrm{~A}-\mathrm{H} 12 \mathrm{~A}$ & 0.9900 \\
\hline $\mathrm{C} 2-\mathrm{H} 2 \mathrm{~B}$ & 0.9900 & $\mathrm{C} 12 \mathrm{~A}-\mathrm{H} 12 \mathrm{~B}$ & 0.9900 \\
\hline $\mathrm{C} 2-\mathrm{C} 3$ & $1.505(7)$ & $\mathrm{O} 4 \mathrm{~B}-\mathrm{C} 9 \mathrm{~B}$ & $1.452(7)$ \\
\hline $\mathrm{C} 3-\mathrm{H} 3 \mathrm{~A}$ & 0.9900 & $\mathrm{O} 4 \mathrm{~B}-\mathrm{C} 12 \mathrm{~B}$ & $1.468(7)$ \\
\hline C $3-\mathrm{H} 3 \mathrm{~B}$ & 0.9900 & C9B-H9BA & 0.9900 \\
\hline $\mathrm{C} 3-\mathrm{C} 4$ & $1.506(6)$ & C9B-H9BB & 0.9900 \\
\hline $\mathrm{C} 4-\mathrm{H} 4 \mathrm{~A}$ & 0.9900 & $\mathrm{C} 9 \mathrm{~B}-\mathrm{C} 10 \mathrm{~B}$ & $1.510(9)$ \\
\hline $\mathrm{C} 4-\mathrm{H} 4 \mathrm{~B}$ & 0.9900 & $\mathrm{C} 10 \mathrm{~B}-\mathrm{H} 10 \mathrm{C}$ & 0.9900 \\
\hline $\mathrm{C} 5-\mathrm{H} 5 \mathrm{~A}$ & 0.9900 & C10B-H10D & 0.9900 \\
\hline $\mathrm{C} 5-\mathrm{H} 5 \mathrm{~B}$ & 0.9900 & $\mathrm{C} 10 \mathrm{~B}-\mathrm{C} 11 \mathrm{~B}$ & $1.497(9)$ \\
\hline $\mathrm{C} 5-\mathrm{C} 6$ & $1.499(6)$ & $\mathrm{C} 11 \mathrm{~B}-\mathrm{H} 11 \mathrm{C}$ & 0.9900 \\
\hline C6-H6A & 0.9900 & $\mathrm{C} 11 \mathrm{~B}-\mathrm{H} 11 \mathrm{D}$ & 0.9900 \\
\hline C6-H6B & 0.9900 & $\mathrm{C} 11 \mathrm{~B}-\mathrm{C} 12 \mathrm{~B}$ & $1.510(8)$ \\
\hline $\mathrm{C} 6-\mathrm{C} 7$ & $1.487(7)$ & $\mathrm{C} 12 \mathrm{~B}-\mathrm{H} 12 \mathrm{C}$ & 0.9900 \\
\hline C7-H7A & 0.9900 & $\mathrm{C} 12 \mathrm{~B}-\mathrm{H} 12 \mathrm{D}$ & 0.9900 \\
\hline $\mathrm{C} 7-\mathrm{H} 7 \mathrm{~B}$ & 0.9900 & & \\
\hline $\mathrm{Cl} 1-\mathrm{Ti} 1-\mathrm{Cl} 2$ & $165.98(5)$ & $\mathrm{H} 7 \mathrm{~A}-\mathrm{C} 7-\mathrm{H} 7 \mathrm{~B}$ & 108.7 \\
\hline $\mathrm{O} 1-\mathrm{Ti} 1-\mathrm{Cl} 1$ & $97.47(9)$ & $\mathrm{C} 8-\mathrm{C} 7-\mathrm{C} 6$ & $106.3(4)$ \\
\hline $\mathrm{O} 1-\mathrm{Ti} 1-\mathrm{Cl} 2$ & $96.55(9)$ & $\mathrm{C} 8-\mathrm{C} 7-\mathrm{H} 7 \mathrm{~A}$ & 110.5 \\
\hline $\mathrm{O} 1-\mathrm{T} 11-\mathrm{O} 2$ & $95.20(13)$ & $\mathrm{C} 8-\mathrm{C} 7-\mathrm{H} 7 \mathrm{~B}$ & 110.5 \\
\hline $\mathrm{O} 1-\mathrm{Ti1}-\mathrm{O} 3$ & $179.21(13)$ & $\mathrm{O} 3-\mathrm{C} 8-\mathrm{C} 7$ & $107.3(4)$ \\
\hline $\mathrm{O} 1-\mathrm{Ti} 1-\mathrm{O} 4 \mathrm{~A}$ & $95.86(13)$ & $\mathrm{O} 3-\mathrm{C} 8-\mathrm{H} 8 \mathrm{~A}$ & 110.3 \\
\hline O1-Ti1-O4B & $95.86(13)$ & $\mathrm{O} 3-\mathrm{C} 8-\mathrm{H} 8 \mathrm{~B}$ & 110.3 \\
\hline $\mathrm{O} 2-\mathrm{Ti} 1-\mathrm{Cl} 1$ & $89.38(8)$ & $\mathrm{C} 7-\mathrm{C} 8-\mathrm{H} 8 \mathrm{~A}$ & 110.3 \\
\hline $\mathrm{O} 2-\mathrm{Ti} 1-\mathrm{Cl} 2$ & $89.04(8)$ & $\mathrm{C} 7-\mathrm{C} 8-\mathrm{H} 8 \mathrm{~B}$ & 110.3 \\
\hline $\mathrm{O} 2-\mathrm{Ti} 1-\mathrm{O} 3$ & $84.79(11)$ & $\mathrm{H} 8 \mathrm{~A}-\mathrm{C} 8-\mathrm{H} 8 \mathrm{~B}$ & 108.5 \\
\hline $\mathrm{O} 2-\mathrm{Ti} 1-\mathrm{O} 4 \mathrm{~A}$ & $168.93(11)$ & $\mathrm{C} 9 \mathrm{~A}-\mathrm{O} 4 \mathrm{~A}-\mathrm{Ti} 1$ & $121.3(7)$ \\
\hline $\mathrm{O} 2-\mathrm{Ti1}-\mathrm{O} 4 \mathrm{~B}$ & $168.93(11)$ & $\mathrm{C} 12 \mathrm{~A}-\mathrm{O} 4 \mathrm{~A}-\mathrm{Ti1}$ & $122.3(10)$ \\
\hline $\mathrm{O} 3-\mathrm{Ti1}-\mathrm{Cl1}$ & $83.32(8)$ & $\mathrm{C} 12 \mathrm{~A}-\mathrm{O} 4 \mathrm{~A}-\mathrm{C} 9 \mathrm{~A}$ & $109.1(11)$ \\
\hline $\mathrm{O} 3-\mathrm{Ti} 1-\mathrm{Cl} 2$ & $82.67(8)$ & O4A-C9A-H9AA & 111.6 \\
\hline $\mathrm{O} 4 \mathrm{~A}-\mathrm{Ti1}-\mathrm{Cl1}$ & $88.68(8)$ & $\mathrm{O} 4 \mathrm{~A}-\mathrm{C} 9 \mathrm{~A}-\mathrm{H} 9 \mathrm{AB}$ & 111.6 \\
\hline
\end{tabular}




\begin{tabular}{|c|c|c|c|}
\hline $\mathrm{O} 4 \mathrm{~A}-\mathrm{Ti} 1-\mathrm{Cl} 2$ & $90.21(8)$ & $\mathrm{O} 4 \mathrm{~A}-\mathrm{C} 9 \mathrm{~A}-\mathrm{C} 10 \mathrm{~A}$ & $100.9(11)$ \\
\hline $\mathrm{O} 4 \mathrm{~A}-\mathrm{Ti} 1-\mathrm{O} 3$ & $84.16(11)$ & $\mathrm{H} 9 \mathrm{AA}-\mathrm{C} 9 \mathrm{~A}-\mathrm{H} 9 \mathrm{AB}$ & 109.4 \\
\hline $\mathrm{O} 4 \mathrm{~B}-\mathrm{Ti1}-\mathrm{Cl1}$ & $88.68(8)$ & $\mathrm{C} 10 \mathrm{~A}-\mathrm{C} 9 \mathrm{~A}-\mathrm{H} 9 \mathrm{AA}$ & 111.6 \\
\hline $\mathrm{O} 4 \mathrm{~B}-\mathrm{Ti} 1-\mathrm{Cl} 2$ & $90.21(8)$ & $\mathrm{C} 10 \mathrm{~A}-\mathrm{C} 9 \mathrm{~A}-\mathrm{H} 9 \mathrm{AB}$ & 111.6 \\
\hline $\mathrm{O} 4 \mathrm{~B}-\mathrm{Ti1}-\mathrm{O} 3$ & $84.16(11)$ & $\mathrm{C} 9 \mathrm{~A}-\mathrm{C} 10 \mathrm{~A}-\mathrm{H} 10 \mathrm{~A}$ & 111.1 \\
\hline Ti1-O1-Ti1 $1^{\mathrm{O}}$ & $179.1(3)$ & $\mathrm{C} 9 \mathrm{~A}-\mathrm{C} 10 \mathrm{~A}-\mathrm{H} 10 \mathrm{~B}$ & 111.1 \\
\hline $\mathrm{C} 1-\mathrm{O} 2-\mathrm{Ti} 1$ & $125.7(2)$ & $\mathrm{H} 10 \mathrm{~A}-\mathrm{C} 10 \mathrm{~A}-\mathrm{H} 10 \mathrm{~B}$ & 109.0 \\
\hline $\mathrm{C} 1-\mathrm{O} 2-\mathrm{C} 4$ & $108.4(3)$ & $\mathrm{C} 11 \mathrm{~A}-\mathrm{C} 10 \mathrm{~A}-\mathrm{C} 9 \mathrm{~A}$ & $103.4(13)$ \\
\hline $\mathrm{C} 4-\mathrm{O} 2-\mathrm{Ti} 1$ & $122.0(2)$ & $\mathrm{C} 11 \mathrm{~A}-\mathrm{C} 10 \mathrm{~A}-\mathrm{H} 10 \mathrm{~A}$ & 111.1 \\
\hline C5-O3-Til & $125.8(2)$ & $\mathrm{C} 11 \mathrm{~A}-\mathrm{C} 10 \mathrm{~A}-\mathrm{H} 10 \mathrm{~B}$ & 111.1 \\
\hline $\mathrm{C} 8-\mathrm{O} 3-\mathrm{Ti} 1$ & $125.6(3)$ & $\mathrm{C} 10 \mathrm{~A}-\mathrm{C} 11 \mathrm{~A}-\mathrm{H} 11 \mathrm{~A}$ & 111.0 \\
\hline $\mathrm{C} 8-\mathrm{O} 3-\mathrm{C} 5$ & $108.5(3)$ & $\mathrm{C} 10 \mathrm{~A}-\mathrm{C} 11 \mathrm{~A}-\mathrm{H} 11 \mathrm{~B}$ & 111.0 \\
\hline $\mathrm{O} 2-\mathrm{C} 1-\mathrm{H} 1 \mathrm{~A}$ & 110.7 & $\mathrm{C} 10 \mathrm{~A}-\mathrm{C} 11 \mathrm{~A}-\mathrm{C} 12 \mathrm{~A}$ & $103.7(13)$ \\
\hline $\mathrm{O} 2-\mathrm{C} 1-\mathrm{H} 1 \mathrm{~B}$ & 110.7 & $\mathrm{H} 11 \mathrm{~A}-\mathrm{C} 11 \mathrm{~A}-\mathrm{H} 11 \mathrm{~B}$ & 109.0 \\
\hline $\mathrm{O} 2-\mathrm{C} 1-\mathrm{C} 2$ & $105.1(4)$ & $\mathrm{C} 12 \mathrm{~A}-\mathrm{C} 11 \mathrm{~A}-\mathrm{H} 11 \mathrm{~A}$ & 111.0 \\
\hline $\mathrm{H} 1 \mathrm{~A}-\mathrm{C} 1-\mathrm{H} 1 \mathrm{~B}$ & 108.8 & $\mathrm{C} 12 \mathrm{~A}-\mathrm{C} 11 \mathrm{~A}-\mathrm{H} 11 \mathrm{~B}$ & 111.0 \\
\hline $\mathrm{C} 2-\mathrm{C} 1-\mathrm{H} 1 \mathrm{~A}$ & 110.7 & $\mathrm{O} 4 \mathrm{~A}-\mathrm{C} 12 \mathrm{~A}-\mathrm{C} 11 \mathrm{~A}$ & $105.5(12)$ \\
\hline $\mathrm{C} 2-\mathrm{C} 1-\mathrm{H} 1 \mathrm{~B}$ & 110.7 & $\mathrm{O} 4 \mathrm{~A}-\mathrm{C} 12 \mathrm{~A}-\mathrm{H} 12 \mathrm{~A}$ & 110.6 \\
\hline $\mathrm{C} 1-\mathrm{C} 2-\mathrm{H} 2 \mathrm{~A}$ & 111.4 & $\mathrm{O} 4 \mathrm{~A}-\mathrm{C} 12 \mathrm{~A}-\mathrm{H} 12 \mathrm{~B}$ & 110.6 \\
\hline $\mathrm{C} 1-\mathrm{C} 2-\mathrm{H} 2 \mathrm{~B}$ & 111.4 & $\mathrm{C} 11 \mathrm{~A}-\mathrm{C} 12 \mathrm{~A}-\mathrm{H} 12 \mathrm{~A}$ & 110.6 \\
\hline $\mathrm{C} 1-\mathrm{C} 2-\mathrm{C} 3$ & $101.9(4)$ & $\mathrm{C} 11 \mathrm{~A}-\mathrm{C} 12 \mathrm{~A}-\mathrm{H} 12 \mathrm{~B}$ & 110.6 \\
\hline $\mathrm{H} 2 \mathrm{~A}-\mathrm{C} 2-\mathrm{H} 2 \mathrm{~B}$ & 109.3 & $\mathrm{H} 12 \mathrm{~A}-\mathrm{C} 12 \mathrm{~A}-\mathrm{H} 12 \mathrm{~B}$ & 108.8 \\
\hline $\mathrm{C} 3-\mathrm{C} 2-\mathrm{H} 2 \mathrm{~A}$ & 111.4 & $\mathrm{C} 9 \mathrm{~B}-\mathrm{O} 4 \mathrm{~B}-\mathrm{Ti1}$ & $130.2(4)$ \\
\hline $\mathrm{C} 3-\mathrm{C} 2-\mathrm{H} 2 \mathrm{~B}$ & 111.4 & $\mathrm{C} 9 \mathrm{~B}-\mathrm{O} 4 \mathrm{~B}-\mathrm{C} 12 \mathrm{~B}$ & $107.6(5)$ \\
\hline $\mathrm{C} 2-\mathrm{C} 3-\mathrm{H} 3 \mathrm{~A}$ & 111.3 & $\mathrm{C} 12 \mathrm{~B}-\mathrm{O} 4 \mathrm{~B}-\mathrm{Ti1}$ & $120.4(4)$ \\
\hline $\mathrm{C} 2-\mathrm{C} 3-\mathrm{H} 3 \mathrm{~B}$ & 111.3 & $\mathrm{O} 4 \mathrm{~B}-\mathrm{C} 9 \mathrm{~B}-\mathrm{H} 9 \mathrm{BA}$ & 111.3 \\
\hline $\mathrm{C} 2-\mathrm{C} 3-\mathrm{C} 4$ & $102.3(4)$ & $\mathrm{O} 4 \mathrm{~B}-\mathrm{C} 9 \mathrm{~B}-\mathrm{H} 9 \mathrm{BB}$ & 111.3 \\
\hline $\mathrm{H} 3 \mathrm{~A}-\mathrm{C} 3-\mathrm{H} 3 \mathrm{~B}$ & 109.2 & $\mathrm{O} 4 \mathrm{~B}-\mathrm{C} 9 \mathrm{~B}-\mathrm{C} 10 \mathrm{~B}$ & $102.5(5)$ \\
\hline $\mathrm{C} 4-\mathrm{C} 3-\mathrm{H} 3 \mathrm{~A}$ & 111.3 & $\mathrm{H} 9 \mathrm{BA}-\mathrm{C} 9 \mathrm{~B}-\mathrm{H} 9 \mathrm{BB}$ & 109.2 \\
\hline $\mathrm{C} 4-\mathrm{C} 3-\mathrm{H} 3 \mathrm{~B}$ & 111.3 & $\mathrm{C} 10 \mathrm{~B}-\mathrm{C} 9 \mathrm{~B}-\mathrm{H} 9 \mathrm{BA}$ & 111.3 \\
\hline $\mathrm{O} 2-\mathrm{C} 4-\mathrm{C} 3$ & $105.7(3)$ & $\mathrm{C} 10 \mathrm{~B}-\mathrm{C} 9 \mathrm{~B}-\mathrm{H} 9 \mathrm{BB}$ & 111.3 \\
\hline $\mathrm{O} 2-\mathrm{C} 4-\mathrm{H} 4 \mathrm{~A}$ & 110.6 & $\mathrm{C} 9 \mathrm{~B}-\mathrm{C} 10 \mathrm{~B}-\mathrm{H} 10 \mathrm{C}$ & 111.1 \\
\hline $\mathrm{O} 2-\mathrm{C} 4-\mathrm{H} 4 \mathrm{~B}$ & 110.6 & $\mathrm{C} 9 \mathrm{~B}-\mathrm{C} 10 \mathrm{~B}-\mathrm{H} 10 \mathrm{D}$ & 111.1 \\
\hline $\mathrm{C} 3-\mathrm{C} 4-\mathrm{H} 4 \mathrm{~A}$ & 110.6 & $\mathrm{H} 10 \mathrm{C}-\mathrm{C} 10 \mathrm{~B}-\mathrm{H} 10 \mathrm{D}$ & 109.1 \\
\hline $\mathrm{C} 3-\mathrm{C} 4-\mathrm{H} 4 \mathrm{~B}$ & 110.6 & $\mathrm{C} 11 \mathrm{~B}-\mathrm{C} 10 \mathrm{~B}-\mathrm{C} 9 \mathrm{~B}$ & $103.2(6)$ \\
\hline $\mathrm{H} 4 \mathrm{~A}-\mathrm{C} 4-\mathrm{H} 4 \mathrm{~B}$ & 108.7 & $\mathrm{C} 11 \mathrm{~B}-\mathrm{C} 10 \mathrm{~B}-\mathrm{H} 10 \mathrm{C}$ & 111.1 \\
\hline $\mathrm{O} 3-\mathrm{C} 5-\mathrm{H} 5 \mathrm{~A}$ & 110.8 & $\mathrm{C} 11 \mathrm{~B}-\mathrm{C} 10 \mathrm{~B}-\mathrm{H} 10 \mathrm{D}$ & 111.1 \\
\hline $\mathrm{O} 3-\mathrm{C} 5-\mathrm{H} 5 \mathrm{~B}$ & 110.8 & $\mathrm{C} 10 \mathrm{~B}-\mathrm{C} 11 \mathrm{~B}-\mathrm{H} 11 \mathrm{C}$ & 110.9 \\
\hline $\mathrm{O} 3-\mathrm{C} 5-\mathrm{C} 6$ & $104.7(4)$ & $\mathrm{C} 10 \mathrm{~B}-\mathrm{C} 11 \mathrm{~B}-\mathrm{H} 11 \mathrm{D}$ & 110.9 \\
\hline $\mathrm{H} 5 \mathrm{~A}-\mathrm{C} 5-\mathrm{H} 5 \mathrm{~B}$ & 108.9 & $\mathrm{C} 10 \mathrm{~B}-\mathrm{C} 11 \mathrm{~B}-\mathrm{C} 12 \mathrm{~B}$ & $104.2(6)$ \\
\hline $\mathrm{C} 6-\mathrm{C} 5-\mathrm{H} 5 \mathrm{~A}$ & 110.8 & $\mathrm{H} 11 \mathrm{C}-\mathrm{C} 11 \mathrm{~B}-\mathrm{H} 11 \mathrm{D}$ & 108.9 \\
\hline $\mathrm{C} 6-\mathrm{C} 5-\mathrm{H} 5 \mathrm{~B}$ & 110.8 & $\mathrm{C} 12 \mathrm{~B}-\mathrm{C} 11 \mathrm{~B}-\mathrm{H} 11 \mathrm{C}$ & 110.9 \\
\hline $\mathrm{C} 5-\mathrm{C} 6-\mathrm{H} 6 \mathrm{~A}$ & 111.1 & $\mathrm{C} 12 \mathrm{~B}-\mathrm{C} 11 \mathrm{~B}-\mathrm{H} 11 \mathrm{D}$ & 110.9 \\
\hline $\mathrm{C} 5-\mathrm{C} 6-\mathrm{H} 6 \mathrm{~B}$ & 111.1 & $\mathrm{O} 4 \mathrm{~B}-\mathrm{C} 12 \mathrm{~B}-\mathrm{C} 11 \mathrm{~B}$ & $106.3(6)$ \\
\hline $\mathrm{H} 6 \mathrm{~A}-\mathrm{C} 6-\mathrm{H} 6 \mathrm{~B}$ & 109.0 & $\mathrm{O} 4 \mathrm{~B}-\mathrm{C} 12 \mathrm{~B}-\mathrm{H} 12 \mathrm{C}$ & 110.5 \\
\hline $\mathrm{C} 7-\mathrm{C} 6-\mathrm{C} 5$ & $103.5(4)$ & $\mathrm{O} 4 \mathrm{~B}-\mathrm{C} 12 \mathrm{~B}-\mathrm{H} 12 \mathrm{D}$ & 110.5 \\
\hline $\mathrm{C} 7-\mathrm{C} 6-\mathrm{H} 6 \mathrm{~A}$ & 111.1 & $\mathrm{C} 11 \mathrm{~B}-\mathrm{C} 12 \mathrm{~B}-\mathrm{H} 12 \mathrm{C}$ & 110.5 \\
\hline $\mathrm{C} 7-\mathrm{C} 6-\mathrm{H} 6 \mathrm{~B}$ & 111.1 & $\mathrm{C} 11 \mathrm{~B}-\mathrm{C} 12 \mathrm{~B}-\mathrm{H} 12 \mathrm{D}$ & 110.5 \\
\hline
\end{tabular}


C6-C7-H7A $\quad 110.5$

$\mathrm{C} 6-\mathrm{C} 7-\mathrm{H} 7 \mathrm{~B}$

Symmetry code: (i) $-x+1, y,-z+3 / 2$.
110.5

$\mathrm{H} 12 \mathrm{C}-\mathrm{C} 12 \mathrm{~B}-\mathrm{H} 12 \mathrm{D}$

108.7 